\title{
INVERSION OF SECOND ORDER DIFFERENTIAL OPERATORS
}

\author{
WALTER RUDIN
}

1. We consider the differential equation

$$
L y(t) \equiv y^{\prime \prime}(t)+p(t) y^{\prime}(t)+q(t) y(t)=0 \quad(a \leqq t \leqq b),
$$

in which $p$ and $q$ are continuous for $a \leqq t \leqq b$, with the boundary conditions

$$
y(a)=y(b)=0 .
$$

We further suppose that (1.1) and (1.2) are incompatible, that is, if $y$ satisfies (1.1) and (1.2), then $y \equiv 0$. Let $\alpha(t), \beta(t)$ be nontrivial solutions of (1.1) such that $\alpha(a)=0, \beta(b)=0$, and construct the Green's function

$$
K(x, t)= \begin{cases}\beta(x) \alpha(t) / W(t) & (a \leqq t \leqq x), \\ \alpha(x) \beta(t) / W(t) & (x \leqq t \leqq b),\end{cases}
$$

where $W=\beta \alpha^{\prime}-\alpha \beta^{\prime}$ is the Wronskian of $\alpha$ and $\beta$.

If $f$ is continuous on $[a, b]$, it is well known that the equation $L F=f$ with the boundary conditions $F(a)=F(b)=0$ is solved by

$$
F(x)=-\int_{a}^{b} K(x, t) f(t) d t .
$$

In the present paper the following question is considered: Under what conditions can a function $F$ be represented in the form (1.4)?

Before stating our result, we generalize the operator $L$.

2. Let $F(t)$ be defined in a neighborhood of a point $x$ in $(a, b)$. There exists a number $\eta>0$ such that any two zeros of any nontrivial solution of (1.1) differ by more than $2 \eta\left[1\right.$, p. 227]. ${ }^{1}$ In what follows, $\eta$ will always have this meaning, and $h$ will always denote a number in $(0, \eta)$. Now, if $a<x-h<x+h<b$, our choice of $\eta$ assures us that there is a unique solution $y(t)=y(t ; F, h)$ of (1.1) such that

$$
y(x+h)=F(x+h), \quad y(x-h)=F(x-h) .
$$

We put

$$
\Delta_{h} F(x)=y(x ; F, h)-F(x),
$$

Presented to the Society, September 4, 1951; received by the editors January 23, 1951.

${ }^{1}$ Numbers in brackets refer to the references cited at the end of the paper. 
and define

$$
\Lambda F(x)=\lim _{h \rightarrow 0} 2 \Delta_{h} F(x) / h^{2},
$$

provided the limit exists. $\Lambda^{*} F(x)$ and $\Lambda_{*} F(x)$ are defined similarly, with lim sup and lim inf in place of lim.

If we choose two solutions $u(t)$ and $v(t)$ of (1.1) such that $u(x)=1$, $u^{\prime}(x)=0, v(x)=0, v^{\prime}(x)=1$, and express $y(t ; F, h)$ in terms of $u(t)$ and $v(t)$, we obtain

$$
\Delta_{h} F(x)=\frac{v(x+h) F(x-h)-v(x-h) F(x+h)}{v(x+h) u(x-h)-v(x-h) u(x+h)}-F(x) .
$$

If $|F(x \pm h)-F(x)|=O(h)$ as $h \rightarrow 0$, we apply Taylor's theorem to $u$ and $v ;(2.3)$ then becomes

$$
\begin{aligned}
\Delta_{h} F(x) & =\frac{F(x+h)+F(x-h)-2 F(x)}{2} \\
& +\frac{h}{4} p(x)(F(x+h)-F(x-h)) \\
& +\frac{h^{2}}{2} q(x) F(x)+o\left(h^{2}\right),
\end{aligned}
$$

from which it follows that $\Lambda F(x)=L F(x)$ if $F^{\prime \prime}(x)$ exists. Thus $\Lambda$ generalizes $L$.

(2.5) Definition. We say that the function $f \in H$ in $(a, b)$ if $f$ is measurable in $(a, b)$, and if

$$
\int_{a}^{b}(x-a)(b-x)|f(x)| d x<\infty .
$$

3. We now state our main result.

Theorem. Let $F(x)$ be continuous and bounded for $a<x<b$. Suppose

(i) $\Lambda^{*} F(x)>-\infty, \Lambda_{*} F(x)<+\infty$ in $(a, b)$, except possibly on countable sets $E_{1}$ and $E_{2}$;

(ii) $\lim \sup _{h \rightarrow 0} \Delta_{h} F(x) / h \geqq 0$ on $E_{1}, \lim \inf _{h \rightarrow 0} \Delta_{h} F(x) / h \leqq 0$ on $E_{2}$;

(iii) there exists a function $g \in H$ in $(a, b)$ such that $g(x) \leqq \Lambda^{*} F(x)$ in $(a, b)$.

Then

(A) $F(a+)$ and $F(b-)$ exist;

(D) $F^{\prime}(x)$ is finite for every $x$ in $(a, b)$;

(C) $F^{\prime \prime}(x)$ is finite for almost all $x$ in $(a, b)$;

(D) $L F \in H$ in $(a, b)$; 
(E) for all $x$ in $(a, b)$,

$$
F(x)=-\int_{a}^{b} K(x, t) L F(t) d t+y(x)
$$

where $y$ is a solution of (1.1), with the boundary values $y(a)=F(a+)$, $y(b)=F(b-)$.

This theorem is of a type similar to previous results obtained in [2] and [3], with (1.1) replacing the Laplace equation and the equation $y^{\prime \prime}-\left(1+x^{2}\right) y=0$ respectively. The present result shows that the method developed in the two earlier papers has wider applicability. In particular, we do not require that the Green's function be symmetric, or positive.

In [3] the main features of the argument were perhaps somewhat obscured by the considerable number of asymptotic formulas needed in dealing with the infinite interval. For this reason we restrict ourselves to a bounded interval in this paper, and assume that the differential equation has no singularities at the end points.

4. To have a convenient symbol for integrals of the type (1.4) we define, for $f \in H$ in $(a, b)$,

$$
\Omega f(x)=-\int_{a}^{b} K(x, t) f(t) d t \quad(a \leqq x \leqq b) .
$$

We shall use the following properties of $\Omega$ :

(4.2) $\Omega f(x)$ is continuous on $[a, b]$ and differentiable in $(a, b)$.

(4.3) $L \Omega f(x)=f(x)$ for almost all $x$ in $(a, b)$.

(4.4) If $a<x-h<x+h<b$, and $h<\eta$, then

$$
\Delta_{h} \Omega f(x)=-\int_{x-h}^{x+h} \Delta_{h} K(x, t) f(t) d t .
$$

(4.5) If $f$ is upper semi-continuous at a point $x$ in $(a, b)$, then

$$
\Lambda^{*} \Omega f(x) \leqq f(x)
$$

Continuity of $\Omega f$ is evident if we write $\Omega f$ in the form

$$
\Omega f(x)=-\beta(x) \int_{a}^{x} \frac{\alpha(t)}{W(t)} f(t) d t-\alpha(x) \int_{x}^{b} \frac{\beta(t)}{W(t)} f(t) d t
$$

Using (4.6) to compute $\lim _{z \rightarrow x}(\Omega f(z)-\Omega f(x)) /(z-x)$ for $x$ in $(a, b)$, a simple calculation proves (4.2).

Next, let $E$ be the set of points $x$ in $(a, b)$ for which 


$$
\begin{aligned}
& \frac{d}{d x} \int_{a}^{x} \frac{\alpha(t)}{W(t)} f(t) d t=\frac{\alpha(x)}{W(x)} f(x), \\
& \frac{d}{d x} \int_{x}^{b} \frac{\beta(t)}{W(t)} f(t) d t=-\frac{\beta(x)}{W(x)} f(x) .
\end{aligned}
$$

Direct differentiation shows that (4.3) holds on $E$.

Since $K(x, t)$ satisfies (1.1) as a function of $x$ for fixed $t, \Delta_{h} K(x, t)$ $=0$ if $|x-t|>h$. (4.4) follows.

(4.3) and (4.4) imply (taking $f \equiv 1$ ),

$$
\lim _{h \rightarrow 0} \frac{2}{h^{2}} \int_{x-h}^{x+h} \Delta_{h} K(x, t) d t=-1 .
$$

Also, it follows from (2.3) that, for $|t-x|<h$,

$$
\Delta_{h} K(x, t)<0 \text {. }
$$

To prove (4.5), choose $m>f(x)$. There exists $\delta>0$ such that $f(t)$ $<m$ for $|t-x|<\delta$. By (4.8),

$$
\Delta_{h} \Omega f(x) \leqq-m \int_{x-h}^{x+h} \Delta_{h} K(x, t) d t \quad(0<h<\delta),
$$

which implies, by (4.7), that $\Lambda^{*} \Omega f(x) \leqq m$. (4.5) follows.

5. Before proceeding to the proof of the main theorem, we define convexity relative to the operator $L$.

(5.1) Definition. The continuous function $F$, defined in $(a, b)$, is said to be $L$-convex in $(a, b)$ if $\Delta_{h} F(x) \geqq 0$ for every $x$ in $(a, b)$ and for every $h<\eta$ such that $a<x-h<x+h<b$.

(5.2) Lemma. If $F$ is continuous in $(a, b)$ and if $\Lambda^{*} F(x) \geqq 0$ in $(a, b)$, except possibly on a countable set $E$ on which $\lim \sup _{h \rightarrow 0} \Delta_{h} F(x) / h$ $\geqq 0$, then $F$ is L-convex in $(a, b)$.

Proof. We suppose first that $\Lambda^{*} F(x)>0$ on the complement of $E$. If $F$ is not $L$-convex, there exists $x_{0}$ in $(a, b)$ and $k<\eta$ such that $\Delta_{k} F\left(x_{0}\right)<0$. Let $y$ be the solution of (1.1) such that $y\left(x_{0}-k\right)$ $=F\left(x_{0}-k\right), \quad y\left(x_{0}+k\right)=F\left(x_{0}+k\right)$. Put $s=F-y$. Then $s\left(x_{0}-k\right)$ $=s\left(x_{0}+k\right)=0$ and $s\left(x_{0}\right)>0$.

We insert the following remark: for each point $x$ in $(a, b)$ there exists at most one solution $\phi(t)$ of (1.1) such that, for some $\delta>0$,

$$
\phi(x)=s(x), \quad \phi(t) \geqq s(t) \text { for }|t-x|<\delta .
$$

For, if $\phi_{1}$ and $\phi_{2}$ satisfy (5.3), and $\phi_{1}^{\prime}(x)-\phi_{2}^{\prime}(x)=2 \epsilon>0$, then $s(t)$ 
$\leqq \min \left(\phi_{1}(t), \phi_{2}(t)\right)$ for $|t-x|<\delta$; and it is not difficult to conclude (using 2.3) that

$$
\limsup _{h \rightarrow 0} \Delta_{h} s(x) / h \leqq-\epsilon<0 .
$$

But this contradicts our hypothesis, since $\Delta_{h} s(x)=\Delta_{h} F(x)$.

Now, choose a point $x_{1}$ in $\left(x_{0}-\eta, x_{0}-k\right)$. There exists a unique solution $\phi$ of (1.1) such that (1) $\phi\left(x_{1}\right)=0$, (2) $\phi(t) \geqq s(t)$ for $t$ in $\left(x_{0}-k, x_{0}+k\right),(3) \phi\left(x_{2}\right)=s\left(x_{2}\right)$ for some $x_{2}$ in $\left(x_{0}-k, x_{0}+k\right)$. Since $E$ is at most countable we can, by the above remark, further restrict $x_{1}$ so that $\phi(t) \neq s(t)$ on $E$ (we merely have to avoid a countable set in $\left.\left(x_{0}-\eta, x_{0}-k\right)\right)$. Having chosen $x_{1}$ in this way, $x_{2} \notin E$, and for small enough $h$,

$$
\Delta_{h} F\left(x_{2}\right)=\Delta_{h} s\left(x_{2}\right) \leqq \Delta_{h} \phi\left(x_{2}\right)=0,
$$

which contradicts $\Lambda^{*} F\left(x_{2}\right)>0$.

In the general case $\left(\Lambda^{*} F \geqq 0\right)$, choose a function $G$ such that $L G$ $\equiv 1$ (for instance, take $G=\Omega f$ with $f \equiv 1$ ), and put $F_{n}=F+G / n$. From the first part of the proof it follows that $F_{n}$ is $L$-convex. Since $F=\lim _{n \rightarrow \infty} F_{n}$ implies $\Delta_{h} F=\lim _{n \rightarrow \infty} \Delta_{h} F_{n}, F$ is $L$-convex.

(5.4) Corollary. If $F$ is continuous in $(a, b)$, and if $\Lambda_{*} F(x) \leqq 0$ $\leqq \Lambda^{*} F(x)$ in $(a, b)$, then $\operatorname{LF}(x)=0$ in $(a, b)$.

(5.5) LemmA. If $F$ is L-convex and bounded above in $(a, b)$, then $\Lambda^{*} F$ and $\Lambda_{*} F \in H$ in $(a, b)$.

Proof. If $F(x)<m$ in $(a, b)$, we subtract a solution $y$ of (1.1) such that $y(a)=y(b)>m$. Taking $F-y$ in place of $F$, we see that we can assume without loss of generality that $F(x)<0$ in $(a, a+\delta)$ and $(b-\delta, b)$ for some $\delta>0$.

Suppose. first that $b-a<2 \eta$. Then $F(x) \leqq 0$ in $(a, b)$ and $K(x, t)$ $>0$ for $x, t$ in $(a, b)$. Consider a sequence $\left\{u_{n}\right\}$ of non-negative upper semi-continuous bounded functions associated with $\Lambda^{*} F$ in $(a, b)$ in the sense of the Vitali-Carathéodory theorem [4, p. 75], and put $U_{n}(x)=F(x)-\Omega u_{n}(x)$ in $(a, b)$. Then, by (4.5),

$$
\Lambda^{*} U_{n}(x) \geqq \Lambda^{*} F(x)-\Lambda^{*} \Omega u_{n}(x) \geqq \Lambda^{*} F(x)-u_{n}(x) \geqq 0 .
$$

Thus $U_{n}$ is $L$-convex in $(a, b)$. Since $\Omega u_{n}(x) \rightarrow 0$ as $x \rightarrow a$ and as $x \rightarrow b$ (by (4.2)), we have $U_{n}(x) \leqq 0$ near the end points, and therefore $U_{n}(x) \leqq 0$ in $(a, b)$. Hence

$$
F(x) \leqq \Omega u_{n}(x) \leqq 0 \quad(a<x<b) .
$$

Since $u_{n} \rightarrow \Lambda^{*} F$ p.p. monotonically, we can pass to the limit as $n \rightarrow \infty$ 
(noting that $K(x, t)>0$ ), and obtain

$$
F(x) \leqq \Omega \Lambda^{*} F(x) \leqq 0 \quad(a<x<b) .
$$

Hence $\Lambda^{*} F \in H$ in $(a, b)$ if $b-a<2 \eta$.

If $b-a \geqq 2 \eta$, the above argument shows that $\Lambda^{*} F$ is summable in every interval of length less than $2 \eta$, and completely interior to $(a, b)$. It follows that $\Lambda^{*} F \in H$ in $(a, b)$.

The lemma is completed by noting that $0 \leqq \Lambda_{*} F(x) \leqq \Lambda^{*} F(x)$ in $(a, b)$.

6. Lemma. If $f \in H$ in $(a, b)$, then there exists a sequence $\left\{u_{n}\right\}$, $n=1,2,3, \cdots$, such that

(1) $u_{n}(x)$ is upper semi-continuous and less than $+\infty$ in $(a, b)$;

(2) $u_{1}(x) \leqq u_{2}(x) \leqq \cdots \leqq f(x)$ in $(a, b)$;

(3) $\lim _{n \rightarrow \infty} u_{n}(x)=f(x)$ p.p. in $(a, b)$;

(4) $u_{n} \in H$ in $(a, b)$.

Proof. Let $\left\{u_{n}^{*}\right\}$ be a sequence of upper semi-continuous functions associated with $(x-a)(b-x) f(x)$ in the sense of the VitaliCaratheodory theorem. The functions $u_{n}(x)=u_{n}^{*}(x) /(x-a)(b-x)$ have the desired properties.

7. Now suppose $F$ satisfies the hypotheses of our theorem. Let $u$ be one of the functions associated with $g$ in the sense of Lemma 6 , and put $U(x)=F(x)-\Omega u(x)$. On the complement of $E_{1},(4.5)$ implies

$$
\Lambda^{*} U(x) \geqq \Lambda^{*} F(x)-u(x) \geqq 0 .
$$

Since $\Omega u$ is differentiable (by (4.2)), (2.4) shows that $\Delta_{h} \Omega u(x)=o(h)$ for $x$ in $(a, b)$, hence $\lim \sup _{h \rightarrow 0} \Delta_{h} U(x) / h \geqq 0$ on $E_{1}$. By (5.2), $U(x)$ is thus $L$-convex in $(a, b)$. Since $U$ is bounded in $(a, b),(5.5)$ and the obvious inequalities

$$
\Lambda_{*} U(x)+\Lambda_{*} \Omega u(x) \leqq \Lambda_{*} F(x) \leqq \Lambda^{*} F(x) \leqq \Lambda^{*} U(x)+\Lambda^{*} \Omega u(x)
$$

show that $\Lambda^{*} F$ and $\Lambda_{*} F \in H$ in $(a, b)$.

Let $f$ be a measurable function defined in $(a, b)$ such that $\Lambda_{*} F(x)$ $\leqq f(x) \leqq \Lambda^{*} F(x)$. Let $\left\{u_{n}\right\}$ be a sequence associated with $f$ in the sense of Lemma 6 , and put $U_{n}(x)=F(x)-\Omega u_{n}(x)$. Proceeding as above, we see that $U_{n}$ is $L$-convex in $(a, b)$. Hence $\Delta_{h} F(x) \geqq \Delta_{h} \Omega u_{n}(x)$ for $x$ in $(a, b), n=1,2,3, \cdots, h$ in $(0, \eta)$. Since $u_{n} \rightarrow f$ p.p. monotonically, we can pass to the limit (taking into account (4.4) and (4.8)), and obtain

$$
\Delta_{h} F(x) \geqq \Delta_{h} \Omega f(x) .
$$

Approximating $f$ similarly by a monotonically decreasing sequence of 
lower semi-continuous functions, we obtain (7.1) with the inequality reversed. Hence

$$
\Delta_{h} F(x)=\Delta_{h} \Omega f(x) .
$$

By (5.4), the function $y(x)=F(x)-\Omega f(x)$ is thus a solution of (1.1). By (4.3), $L F(x)=f(x)$ p.p. in $(a, b)$. This gives us the representation (E) in the conclusion of the theorem.

By (4.2) and (4.3) the other parts of the conclusion are immediate consequences of the representation formula. This completes the proof.

\section{REFERENCES}

1. E. L. Ince, Ordinary differential equations, New York, Dover, 1944.

2. W. Rudin, Integral representation of continuous functions, Trans. Amer. Math. Soc. vol. 68 (1950) pp. 279-287.

3. - Uniqueness theory for Hermite series, Trans. Amer. Math. Soc. vol. 70 (1951) pp. 387-403.

4. S. Saks, Theory of the integral, 2d ed., Warsaw, 1937.

Massachusetts Institute of Technology 